\title{
Erratum to: Immediate Repair of Major Abdominal Wall Defect After Extensive Tumor Excision in Patients With Abdominal Wall Neoplasm: A Retrospective Review of 27 Cases
}

\author{
Rui Tang, MD, $\mathrm{PhD}^{1}$, Yan Gu, MD, $\mathrm{PhD}^{1}$, Ding-Quan Gong, $\mathrm{MD}^{1}$, and Yun-Liang Qian, $\mathrm{MD}^{2}$ \\ ${ }^{1}$ Department of General Surgery, Hernia and Abdominal Wall SurgeryCenter of Shanghai Jiaotong University, Shanghai \\ Ninth People's Hospital, affiliated to Shanghai Jiaotong University, School of Medicine, Shanghai, China; ${ }^{2}$ Department of \\ Plastic Surgery, Shanghai Ninth People's Hospital, affiliated to Shanghai Jiaotong University, School of Medicine, \\ Shanghai, China
}

\section{ERRATUM TO: ANN SURG ONCOL DOI 10.1245/S10434-009-0548-8}

In the article by Tang et al., the title appeared incorrectly. The correct title appears correctly as follows: "Immediate Repair of Major Abdominal Wall Defect After Extensive Tumor Excision in Patients With Abdominal Wall Neoplasm: A Retrospective Review of 27 Cases"

The online version of the original article can be found under doi:10.1245/s10434-009-0548-8.

(C) Society of Surgical Oncology 2009

Published Online: 14 November 2009

Y. Gu, MD, PhD

e-mail: yangu@sjtu.edu.cn 